This item was submitted to Loughborough's Research Repository by the author.

Items in Figshare are protected by copyright, with all rights reserved, unless otherwise indicated.

\title{
A Bayesian Tobit quantile regression approach for naturalistic longitudinal driving capability assessment
}

PLEASE CITE THE PUBLISHED VERSION

https://doi.org/10.1016/j.aap.2020.105779

PUBLISHER

Elsevier

VERSION

AM (Accepted Manuscript)

\section{PUBLISHER STATEMENT}

This paper was accepted for publication in the journal Accident Analysis and Prevention and the definitive published version is available at https://doi.org/10.1016/j.aap.2020.105779

LICENCE

CC BY-NC-ND 4.0

\section{REPOSITORY RECORD}

Yu, Rongjie, Xiaojie Long, Mohammed Quddus, and Junhua Wang. 2020. "A Bayesian Tobit Quantile Regression Approach for Naturalistic Longitudinal Driving Capability Assessment”. Loughborough University. https://hdl.handle.net/2134/13079618.v1. 


\title{
A Bayesian Tobit quantile regression approach for naturalistic longitudinal driving capability assessment
}

\author{
Rongjie Yua , Ph.D., Associate Professor \\ * Corresponding Author \\ E-mail: yurongjie@tongji.edu.cn
}

Xiaojie Longa ${ }^{\text {a }}$ Graduate Research Assistant

E-mail: marlingism@163.com

\author{
Mohammed Quddus ${ }^{\mathrm{b}}$, Ph.D., Professor \\ E-mail: M.A.Quddus@lboro.ac.uk
}

Junhua Wanga , Ph.D., Professor

E-mail: benwjh@163.com

${ }^{\text {a }}$ The Key Laboratory of Road and Traffic Engineering, Ministry of Education, 201804, Shanghai, China

${ }^{\mathrm{b}}$ School of Architecture, Building and Civil Engineering, Loughborough University, Loughborough LE11, 3TU, United Kingdom

Manuscript submitted for potential publication in Accident Analysis \& Prevention 
1 Abstract: Given the severe traffic safety issue, tremendous efforts have been devoted to identify the crash contributing factors for developing and implementing safety improvement countermeasures. According to the study findings, driving behaviors have attributed to the majority crash occurrence, among which inadequate driving capability is a key factor. Therefore, a number of studies have been conducted for developing techniques associated with the driving capability assessment and its various improvement. However, the conventional assessment approaches, such as driving license exams and vehicle insurance quotes, have only focused on basic driving skill evaluations or aggregated driving style classifications, which failed to quantify driving capability from the safety perspective with respect to the complex driving scenarios. In this study, a novel longitudinal driving capacity assessment and ranking approach was developed with naturalistic driving data. Two Responsibility-Sensitive Safety (RSS) based driving capability indicators from the perspectives of risk exposure and severity were first proposed. Then, Bayesian Tobit quantile regression (BTQR) models were introduced to explore the relationships between driving capability indicators with trip level characteristics from the aspects of travel features, operational conditions, and roadway characteristics. The modeling results concluded that nighttime driving and higher average speed would lead to higher longitudinal collision risk and its severity. Besides, the BTQR models have provided varying factors significances among different quantile levels, for instance, driving duration is only significant at high quantiles for the driving capability indicators, implying that duration only affects drivers with large longitudinal risk exposures and strong close following tendencies. Furthermore, the case studies provided how to deploy the developed model to obtain the relative longitudinal driving capability rankings. Finally, the model applications from the aspects of commercial fleet safety management and comparing the autonomous vehicles' longitudinal driving behaviors with human drivers have been discussed.

Keywords: Driving capability assessment; Longitudinal driving safety; ResponsibilitySensitive Safety; Bayesian Tobit quantile regression model; Naturalistic driving data

\section{Introduction}

Traffic safety is currently a growing problem globally. According to the Global Status Report (World Health Organization 2018), the annual traffic-related fatalities had reached a total of 1.35 million worldwide and traffic crash has become the leading cause of death for people aged 15-29 years. Given the critical safety status, identifying and understanding traffic crash contributing factors have become a critical issue and an important research topic. In-depth investigations have shown that human errors have attributed to most traffic crashes. For instance, Singh (2015) concluded that errors 
relating to driving behavior present in almost a $94 \%$ of the crashes, which can be manifested as driver recognition errors (e.g. inattentive driving), decision errors (e.g. aggressive driving, false assumption of others' actions, misjudgment of gap or others' speed) and performance errors (e.g. overcompensation, poor directional control). In addition, driving capability (e.g. driving skill, driving style) was recognized as one of the key influencing factors for the driving behavior errors (Elander et al. 1993). To conclude, tremendous efforts have been invested for the driving capability assessment with the purpose of traffic safety improvement.

Meanwhile, from the perspective of driver management, driving capability assessment has been conducted in different formats given their varying objectives, among which the typical ones are driving license exams and vehicle insurance quotes. During the driving license application process, the transportation departments would organize driving tests for issuing a license through standardized road tests and programed knowledge exams. These road tests featured with dedicated modules, such as approaching a crossing, backing, and following at a safe distance (Florida DMV 2019), which could only be utilized for basic driving skill evaluations since the operating speed is relatively low and limited interactions with traffic participants were considered.

As for vehicle insurance companies, driving capability assessments were mostly being conducted based on empirical vehicle telematics data. Drivers were categorized into subgroups based upon the risky behaviors and driving style analyses. This kind of assessments usually concludes with limited numbers of driver groups (e.g., Johnson and Trivedi (2011) concluded 2 groups with typical and aggressive). However, the unsupervised learning analysis approach holds the drawbacks of lacking either ranking information between groups or direct associations between the grouping results with driver safety status (e.g., crash and violation records shown in (Merrikhpour et al. 2013)). Besides, the insurance companies could only capture the ego-vehicle kinematic data, which failed to consider the interactions within the real-world traffic environments.

With the rapid developments of data collection techniques, naturalistic driving data with enriched traffic environment information (either through video recorders or radar sensors) are the preferred choice for driving performance investigations. Relevant studies have been conducted to evaluate the driving behaviors based on specific scenarios such as car-following (Higgs and Abbas 2013) and the cut-in events (Wang et al. 2019). However, the majority studies employed the naturalistic driving data obtained fragmented data based on strict criteria, which have abandoned a certain proportion of the original data. For instance, Higgs and Abbas (2013) collected 14,500-hour driving data with 735,00 miles traveled but only identifed 392 valid car-following events for their analysis. In order to conduct broader pictures of driving capability assessment, the 
driving task transition periods (e.g., from car-following to lane change) and the risk exposures within the continuous traffic environment (Zheng et al. 2013) are needed to be considered.

Therefore, in this study, a novel trip-level assessment approach is proposed to fill the gaps in driving capability quantification in real-world traffic environments. With a total of 508 trips of streaming naturalistic driving data from Shanghai urban expressway system, comprehensive evaluations of longitudinal driving risk exposures, driving behaviors, and potential collisions would be conducted for each trip, which is also the basic assessment and summary unit for driver management projects (Galkin et al. 2019) and driving assistant apps (e.g., Amap). Besides, since the longitudinal movements is the principal part of vehicle behaviors and rear-end crashes are one of the most frequently occurring types (National Safety Council 2017), longitudinal driving capability based on the safe distance keeping behavior is focused in this study.

The rest of the paper is organized as follows: the second section discusses relevant studies from the perspectives of driving capability indicators and assessment methods. The third section provides the introductions of driving capability indicators and analysis methodology, which are followed by a detailed description of empirical analysis data with preparation. Then, the modeling results and model application case study are presented. And finally, the Discussion and Conclusion of the work are given.

\section{Literature Review}

In this section, existing driving capability assessment indicators and the adopted evaluation techniques have been summarized. In addition, the research gaps that need to be filled were pointed out.

\subsection{Longitudinal safety indicators}

Previous studies have utilized different longitudinal safety surrogate measures to evaluate the safe distance keeping performance. The indicators can be categorized into time-based and distance-based indicators, which are summarized in Table 1, based upon which risky moments or safety critical events can be identified. However, under the streaming data environment, direct summation of the above-mentioned indicators has no physical meanings, which indicates that they cannot be applied to category or grade the trip-level performance of longitudinal driving behaviors. In addition, several studies have taken a step further to evaluate different severity levels and take the impacts of the heterogeneous traffic scenarios into consideration (Tarko 2012, Zheng et al. 2014a). However, the severity classification threshold determination remains to be an unsolved challenge (Zheng et al. 2014b, Mahmud et al. 2017, Johnsson et al. 2018). 


\begin{tabular}{llll}
\hline Dimension & Indicators & Suitability & Limitations \\
\hline Time-based & Time to Collision (TTC) (Van der Horst & & \\
& 1991), Headway (Ohta 1993), Time to & & Not preferable to \\
& $\begin{array}{l}\text { Accident (Hydén 1987), Post- } \\
\text { Encroachment Time (Allen } \text { et al. 1978) }\end{array}$ & $\begin{array}{l}\text { Identification of } \\
\text { risky moments or }\end{array}$ & $\begin{array}{l}\text { use directly for } \\
\text { continuous }\end{array}$ \\
bistance- & Potential Index for Collision with Urgent & safety critical & driving process \\
& Deceleration (Iida et al. 2001), Margin to & events & assessment \\
& Collision (Kitajima et al. 2009), Proportion & & \\
\hline & of Stopping Distance (Allen et al. 1978) & & \\
\hline
\end{tabular}

Furthermore, with the purpose of providing a complete and comprehensive scope of the risk level, two derived TTC based safety indicators were introduced, which are time exposed time-to-collision (TET) and time integrated time-to-collision (TIT) (Minderhoud and Bovy 2001). TET is the duration of exposition to safety-critical TTC values, while TIT expresses the level of safety by integrating the drivers' TTC profiles. Li et al. used TET and TIT to conduct safety evaluation between novice and experienced drivers (Li et al. 2015). However, there are also limitations in TET and TIT, since TTC alone cannot evaluate absolute safety due to the lack of physical meanings. Furthermore, TTC evaluates safety from the perspective of collision rather than driving performance.

Therefore, longitudinal driving capability indicators with considerations of both realistic traffic environments and adaptive to relative capability comparison from driving safety perspective are required further explorations. The ResponsibilitySensitive Safety (RSS) Safe Distance is a new situation-aware collision avoidance strategy for autonomous vehicles (AV) proposed by Mobileye (Shalev-Shwartz et al. 2017), which provides dynamic minimum safe distance thresholds that accommodate to differential traffic environments and meet the requirements of driving safety assessment for naturalistic driving conditions. However, considering RSS is still an instant index that cannot be applied to streaming data conditions for driving capability assessment, extensions of RSS were investigated and introduced later (see section 3.1).

\subsection{Driving capability assessment algorithms}

Among the driving capability assessment studies, varying algorithms, such as comparative analysis, regression models, and unsupervised algorithms, have been utilized. Comparative analysis techniques such as t-test (Stolwyk et al. 2006, Meng and Siren 2012), chi-square test (Meng and Siren 2012) and ANOVA (Merrikhpour et al. 2013, Malhotra et al. 2017) were used to explore driving capability differences between heterogeneous groups or analyze the influencing factors such as aging, diseases and drugs. However, the methods cannot classify the driving capability with ranking 
information. Moreover, although the regression analysis methods benefit with revealing the influencing factors such as driving environment and individual characteristics on driving capability (Ghasemzadeh and Ahmed 2017), as a supervised learning algorithm, prior information of the driving capability category is required. Since driving capability is a relative concept, it is difficult to label the categories due to lack of priori knowledge. In addition, unsupervised algorithms do not require understanding of the underlying process, of which the classification is achieved through statistical analysis of the input metrics inherent to the algorithms. Several studies have used unsupervised algorithms to classify the heterogonous drivers into groups (Sagberg et al. 2015, Martinez et al. 2017). However, this kind of method holds difficulties in quantifying driving ability levels and interpretation.

Recently, quantile regression $(\mathrm{QR})$, which is the extension of linear regression, has attracted increasing attentions in the fields of economics and medical science (Koenker 2017). QR was initially proposed by Koenker and Bassett (1978). Whereas mean regression estimates the conditional mean of the response variable, QR permits to estimate any quantile along the conditional distribution of the response variable and explore the relationships between the quantile locations and covariates (the "quantile" used here has the same meaning with "percentile", e.g. quantile 0.95 is equal to $95^{\text {th }}$ percentile). Besides, the QR estimations are more robust against outliers as well as multimodality (Koenker 2005). QR has been applied to model traffic crash data (Qin 2012, Liu et al. 2013) and identify crash hotspots (Washington et al. 2014). And QR has demonstrated its flexibility of estimating trends at different quantiles and the capability to tackle data with heterogeneity, which would benefit the driving capability assessment.

To sum up, the abovementioned related works showed that there are limitations in the existing longitudinal driving capability evaluation for both the indicators and assessment methods. The employed indicators failed to account for the heterogeneous traffic scenarios and cannot meet the needs of trip-level driving capability ranking. Besides, the assessment algorithms cannot meet the requirements of quantification ranking and influencing factors interpretations. Therefore, in order to fill the abovementioned gaps, streaming-data-applicable indexes based on RSS would be established and QR analysis technique would be developed in this study to reveal a broader and complete view of the relationships between driving capability and trip characteristicsits influencing factors. 


\section{Methodology}

\subsection{RSS-based longitudinal driving capability indicators}

As mentioned in section 2.1, the RSS model could accommodate to different traffic environments and meet the requirements of safety assessment for naturalistic driving conditions. Thus RSS-based driving capability indicators are further investigated and introduced in this section.

The RSS safe longitudinal distance model is set as follows. Assume that there are two consecutive vehicles moving on the same lane in the same direction. The minimum safe longitudinal distance between the front-most point of the ego vehicle and the rearmost point of the leading vehicle (LV) can be described as follows (Shalev-Shwartz et al. 2017):

$$
d_{\min }=\left[v_{e} \rho+\frac{a_{\max , a} \rho^{2}}{2}+\frac{\left(v_{e}+a_{\max , a} \rho\right)^{2}}{2 a_{\min , b}}-\frac{v_{l}^{2}}{2 b_{\max , b}}\right]^{+}
$$

which means that the two vehicles will not collide with each other, if the LV brakes by at most $b_{\max , b}$ until a full stop, and the ego vehicle accelerates by at most $a_{\max , a}$ using the response time $\rho$, and then brakes by at least $a_{\min , b}$ until a full stop. The nomenclatures in (1) are given in Table 2. The parameter values of $a_{\max , a}, a_{\min , b}, b_{\max , b}$ and $\rho$ in this study were calibrated referring to related studies (as shown in Table 2), while the values of $v_{e}$ and $v_{l}$ were observed through the analysis dataset.

Table 2: Recommended parameter values for the RSS model

\begin{tabular}{lll}
\hline Symbol & Definition & Value \\
\hline$d_{\text {min }}$ & $\begin{array}{l}\text { the minimum safe longitudinal distance between ego } \\
\text { vehicle and } \mathrm{LV} /(\mathrm{m} / \mathrm{s}), \text { referred to as RSS threshold }\end{array}$ & \\
$v_{e}$ & the initial speed of ego vehicle $/(\mathrm{m} / \mathrm{s})$ & $\begin{array}{l}\text { Available from } \\
\text { naturalistic driving data }\end{array}$ \\
$v_{l}$ & the initial speed of $\mathrm{LV} /(\mathrm{m} / \mathrm{s})$ & \\
$a_{\text {max }, a}$ & the maximum acceleration rate of ego vehicle $/\left(\mathrm{m} / \mathrm{s}^{2}\right)$ & $0.2 \mathrm{~g}(\mathrm{Li}$ et al., 2018) \\
$a_{\text {min, }, b}$ & the minimum deceleration rate of ego vehicle $/\left(\mathrm{m} / \mathrm{s}^{2}\right)$ & $0.3 \mathrm{~g}$ (Loeb et al., 2015) \\
$b_{\text {max }, b}$ & the maximum deceleration rate of $\mathrm{LV} /\left(\mathrm{m} / \mathrm{s}^{2}\right)$ & $0.4 \mathrm{~g}$ (Bergasa et al., 2014) \\
$\rho$ & the response time lag $/(\mathrm{s})$ & 1 (Bergasa et al., 2014) \\
\hline
\end{tabular}


Fig. 1 is a space-time diagram which shows the longitudinal distance profile on an urban expressway driving segment where the red line represents $d$ (the relative distance to the LV) and the green line represents $d_{\min }$ (the RSS threshold). At instant T0, a LV (LV1) is detected. Before instant T1, the ego vehicle approaches the LV with the relative distances closer than $d_{\min }$. Between T1 to T2, EV manages to adjust the vehicle speed and keep the longitudinal distances swinging with small amplitudes near dynamic RSS thresholds. At a certain moment T2, there is a sudden drop of $d$ due to a cut-in vehicle (LV2). The speed of EV has to increase the gap in order to maintain a safe distance. At T3, EV reaches the RSS threshold and then operates at longitudinal safe distances to a LV. From Fig. 1, it can be seen that the RSS thresholds vary substantially during the driving. In addition, the driver's longitudinal distance keeping behavior exhibits oscillation features, with changes in speed in order to maintain a safe distance. If the RSS threshold $d_{\text {min }}$ is applied to distinguish safe and safety-critical approach situations, such longitudinal distance profiles can be employed in the determination of safety-critical exposition distribution to derive driver capability indicators.

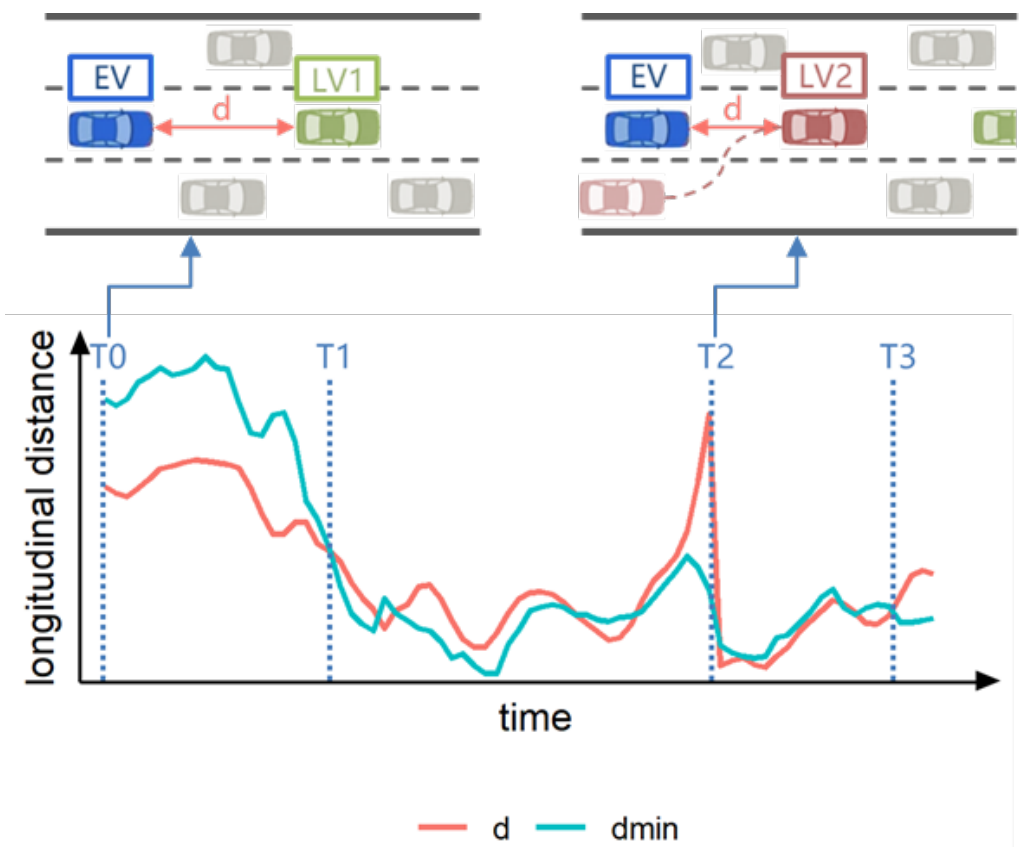

Fig. 1. Example longitudinal distance profile

In this study, two RSS-based longitudinal driving capability indicators (denoted as $p_{\text {exposure }}$ and $\left.p_{\text {severity }}\right)$ were developed. Suppose that $d$ and $d_{\min }$ values at an instant $t_{i}$ do not change during a small time step $\tau_{i}$. For the considered time duration $t$, the 
indicators are therefore as follows:

$$
\begin{aligned}
& p_{\text {exposure }}=\frac{\text { exposure }}{t}=\frac{\sum_{i} \delta_{i} \times \tau_{i}}{t}, \delta_{i}=\left\{\begin{array}{l}
1, \forall 0 \leq d\left(t_{i}\right) \leq d_{\text {min }}\left(t_{i}\right) \\
0, \text { else }
\end{array}\right. \\
& p_{\text {severity }}=\frac{\text { severity }}{\text { exposure }}=\frac{\sum_{i} \frac{d_{\min }\left(t_{i}\right)-d\left(t_{i}\right)}{d_{\min }\left(t_{i}\right)} \delta_{i} \times \tau_{i}}{\sum_{i} \delta_{i} \times \tau_{i}}
\end{aligned}
$$

The first indicator is termed as the longitudinal risk exposure, denoted as $p_{\text {exposure }}$, which is the proportion to the trip duration when the ego vehicle encounters a safetycritical situation (defined as a moment with $d<d_{\min }$ ). The calculation of $p_{\text {exposure }}$ is shown in Lemma (2), where exposure is the summation of all moments that a driver approaches to a LV with $d<d_{\min }$ (as shown in Fig. 2). Thus, a lower $p_{\text {exposure }}$ value indicates that the driver tends to keep a longer distance from the LV during a trip.

However, the entity of the longitudinal distance lower than the safety threshold is also closely related to collision risk which cannot be evaluated by the exposure indicator alone. Therefore, in order to take the impact of the $d_{\min }$ values into account in the assessment, the longitudinal risk exposure indicator, denoted as $p_{\text {severity }}$, is developed, which is the ratio of severity and exposure, as shown in Lemma (3). The severity notion is the summation of the ratio of the gap between the observed relative distance and RSS threshold when an ego vehicle encounters a safety critical situation over a trip, as shown in Fig. 2. Therefore, $p_{\text {severity }}$ can be seen as the average severity, which expresses the aggregated risk level of safety critical events within a trip. The lower the $p_{\text {severity }}$ values, the closer the gap between realistic distance and the minimum safe longitudinal distance when the former is smaller, thus the safer the situation. In addition, it can be seen that the ranges of both indicators are from 0 to 1 . 


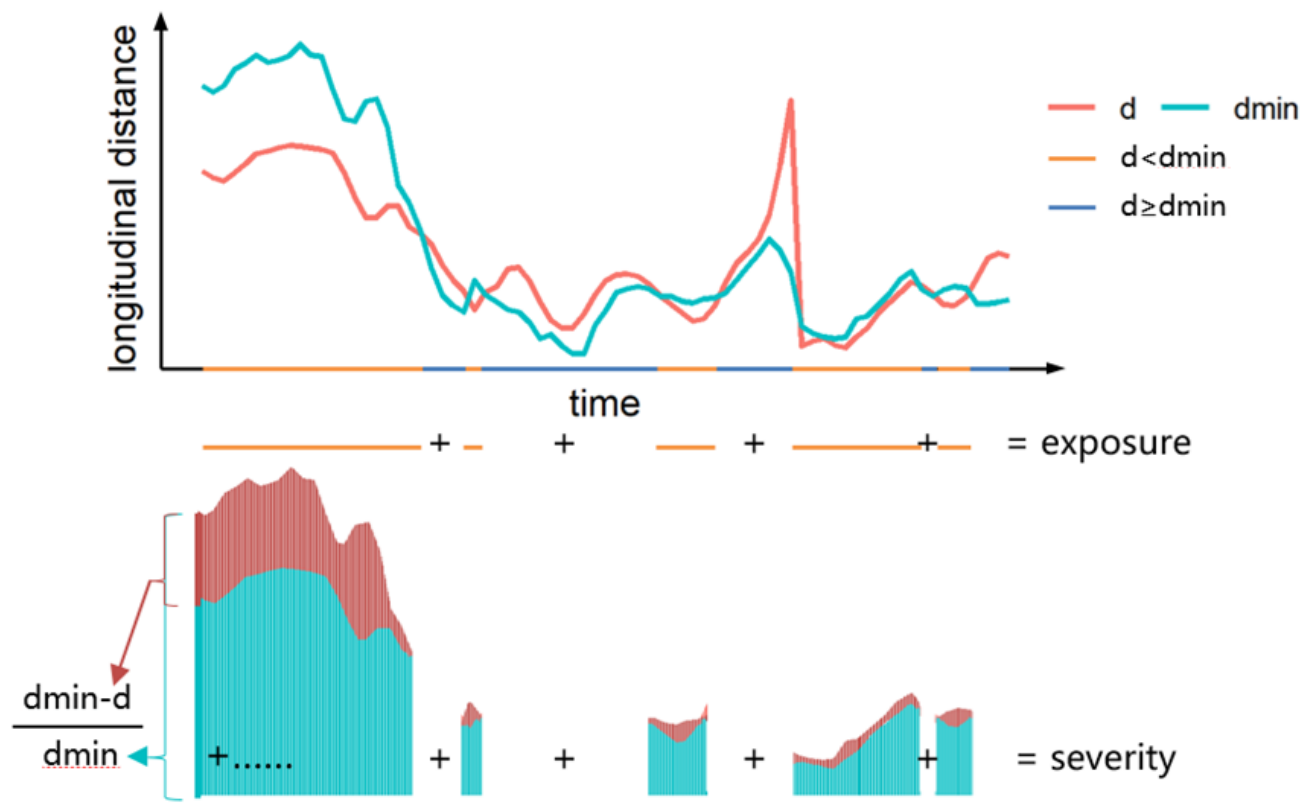

Fig. 2. Example longitudinal distance profile for driving capability indicator calculation

\subsection{Bayesian Tobit quantile regression models}

In order to obtain the driving capability ranking information and simultaneously considering the varying driving conditions of the observed sampled data, QR modeling technique was employed here. Since the longitudinal driving capability indicators $p_{\text {exposure }}$ and $p_{\text {severity }}$ are both continuous values ranging from 0 to 1 , the ordinary $\mathrm{QR}$ modeling method may lead to biased estimations (Yu and Stander 2007). The Tobit model, which was initially proposed by Tobin (Tobin 1958), is employed to address the censored dependent variable issue. Censoring represents the limitation that can result in a data aggregation at a lower threshold (left-censored) or upper threshold (rightcensored) or both. As for $p_{\text {exposure }}$ and $p_{\text {severity }}$, the data are left-censored at 0 and rightcensored at 1 . Thus, the Tobit model could be described as follows:

$$
y_{i}^{*}=x_{i}^{\prime} \beta+\varepsilon_{i}, \quad i=1, \ldots, n, \quad \varepsilon_{i}^{i i d} \square N\left(0, \sigma^{2}\right)
$$

where $x_{i}=\left(\right.$ Duration $_{i}$, Peak $_{i}$, Night $_{i}$, Avg.Speed $_{i}$, Std.Speed $_{i}$, Road $\left._{i}\right)$ is a vector of explanatory variables, $\beta$ is a vector of regression coefficients, $\varepsilon_{i}$ is subject to an identically independent normal distribution with mean zero and variance $\sigma^{2}$. The observed longitudinal driving capability indicator $y_{i}\left(p_{\text {exposure }}\right.$ or $\left.p_{\text {severity }}\right)$ is supposed to be related to the latent variable by the following formulation: 


$$
y_{i}= \begin{cases}1, & y_{i}^{*}>1 \\ y_{i}^{*}, & 0 \leqslant y_{i}^{*} \leqslant 1 \\ 0, & y_{i}^{*}<0\end{cases}
$$

1 Then, in view of the $p$-th quantile regression model for $y_{i}^{*}$ :

$$
y_{i}^{*}=x_{i}^{\prime} \beta_{p}+\varepsilon_{i}
$$

2 the $p$-th quantile regression model of $y_{i}^{*}$ can be expressed as

$$
Q_{y_{i}^{*} \mid x_{i}, \beta_{p}}(p)=x_{i} \beta_{p}
$$

3 where $Q_{y_{i}^{*} \mid x_{i}, \beta_{p}}(\cdot)$ stands for the conditional quantile function. If $p=0.5$,

$4 Q_{y_{i}^{*} \mid x_{i}, \beta_{p}}(0.5)$ is the conditional median.

5 The TQR estimates $\hat{\beta}_{p}$ can be described as the following problem:

$$
\hat{\beta}_{p}=\arg \min \sum_{i=1}^{n} \rho_{p}\left(y_{i}^{*}-x_{i} \beta_{p}\right), \text { where } \beta_{p}(x)= \begin{cases}x p, & x \geq 0 \\ x(p-1), & x<0\end{cases}
$$

6 For any $p \in(0,1)$ the loss function $\rho_{p}$ assigns a weight of $p$ to positive residuals

7 and a weight of $(1-p)$ to negative residuals. The $p$-th regression quantile coincides

8 with the maximum likelihood estimate under independent asymmetric Laplace distribution (ALD) for the unobserved error terms, which is needed for the specification of the likelihood in the Bayesian framework.

Then, in order to implement Bayesian quantile inference, the three-parameter ALD with a skewness parameter is employed to model the quantile of interest (Yu and Stander, 2007), whose probability density function is given by:

$$
f(x \mid \mu, \sigma, p)=\frac{p(1-p)}{\sigma} \exp \left\{-\rho_{p}\left(\frac{x-\mu}{\sigma}\right)\right\}
$$

where $\mu$ is the location parameter. to minimize Lemma (6) is equivalent to maximize the regression likelihood of Lemma (7) utilizing ALD errors with $\mu=x_{i} \beta_{p}$.

In order to compute the regression coefficients, a full Bayesian inference using the Gibbs sampling method is implemented to construct the model. Gibbs sampling is a commonly used means of Bayesian inference because of its tractability and efficiency (Yu and Stander 2007, Yue and Hong 2012). For each model, the Bayesian estimates 
are obtained based on 15,000 MCMC iterations with 5,000 burn-in and one thin.

Different from the $95 \%$ confidence interval of maximum likelihood estimation, the results present Bayesian credible intervals (BCI) as a probability statement about the parameter itself; i.e., a 95\% BCI contains the true parameter value with $95 \%$ certainty. If the $95 \% \mathrm{BCI}$ of the posterior mean does not include 0 , it implies that this effect is statistically significant at the 95\% level. Besides, the Deviance Information Criteria (DIC) was used as the criterion to compare the model goodness-of-fits. Generally, the lower the DIC, the better the model fits. And it is suggested that differences in DIC of lower than 5 considered equally well, differences between 5 and 10 considered substantial, while differences greater than 10 indicate less support to the model with higher DIC (Spiegelhalter et al. 2002). In this study, estimation and computations for Bayesian Tobit quantile regression (BTQR) and Bayesian Tobit mean regression (BTR) are performed in R with package "Brq" (Alhamzawi 2018) and "MCMCpack" (Martin et al. 2011) respectively.

\section{Empirical Data}

For the purpose of trip-level driving capability assessment under streaming data environment, naturalistic driving data with three types of data collected at a rate of $1 \mathrm{~Hz}$ were utilized here, which consist of location information from Global Positioning Systems (GPS), vehicle kinematic characteristics (e.g. speed, longitudinal and lateral acceleration) from speedometers and accelerometers, and surrounding vehicles' operation data (e.g. surrounding vehicles with relative speeds, accelerations and distances referred to ego vehicle) captured by the radar system installed on the vehicle's front bumper. Moreover, in order to neutralize the influences of signalization control, interactions with pedestrians and non-motor vehicles on longitudinal driving behavior, the urban expressway driving data were utilized for the empirical analysis.

As for data processing, first, with the help of an Open Source Routing Machine (ORSM) based map-matching tool (Mapbox 2018), GPS data can be matched with geographic and road information (e.g. road name, road type) and data from Shanghai urban expressway network (as shown in Fig. 3) were filtered. In addition, in order to obtain reliable driving performance, a minimum 5-minute expressway driving duration was set for the sampled trips. And a total of 508 expressway driving trips were extracted that represented about $3,893 \mathrm{~km}$ of expressway driving data. Then, the vehicle kinematic characteristics and the LV (the vehicle just in front of the ego vehicle information) were extracted. Trip-level characteristics including travel, operational, and roadway characteristics along with longitudinal driving capability indicators were recorded as summarized in Table 3. 


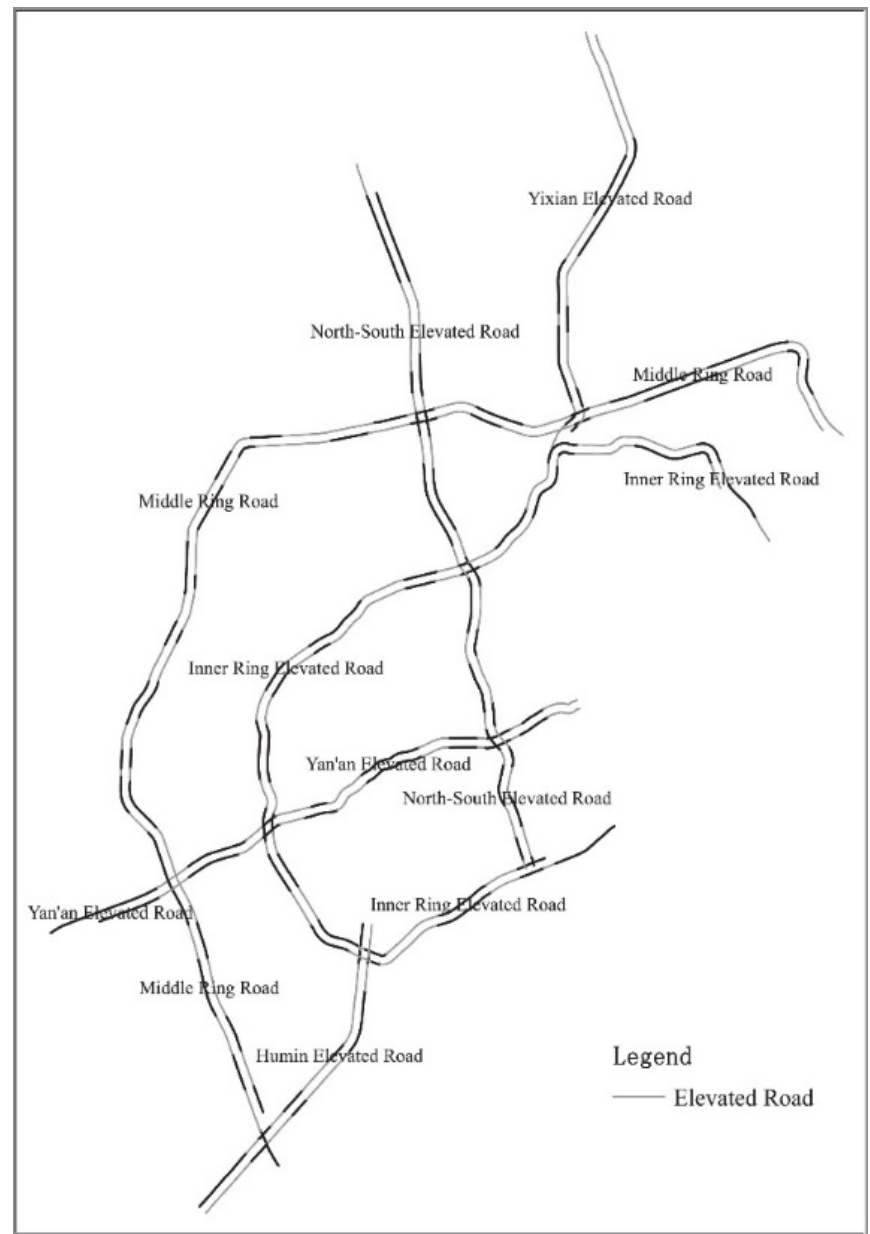

Fig. 3. Shanghai urban expressway network

3

4

5

6

7

8

9

10

11

12

13

14

15 


\begin{tabular}{|c|c|c|c|}
\hline Variable & Definition & Mean & Std. Dev. \\
\hline \multicolumn{4}{|c|}{ Longitudinal driving capability indicators } \\
\hline$p_{\text {exposure }}$ & $\begin{array}{l}\text { The proportion to the trip duration when the ego vehicle } \\
\text { encounters a safety-critical situation (defined as a moment }\end{array}$ & 0.656 & 0.175 \\
\hline & with $\left.d<d_{\min }\right)$ & & \\
\hline$p_{\text {severity }}$ & The average value of the ratio of $d_{\min }-d$ and $d_{\min }$ when & 0.271 & 0.118 \\
\hline & ego vehicle encounters a safety-critical situation & & \\
\hline \multicolumn{4}{|c|}{ Travel features } \\
\hline Duration & Driving duration on the expressway system/(h) & 0.194 & 0.084 \\
\hline Peak & $\begin{array}{l}\text { 0: off-peak, 1: peak (based on average speeds and volumes } \\
\text { of different time period, peak hours for the expressway } \\
\text { system were identified as } 7 \text { a.m. to } 9 \text { a.m and } 4 \text { p.m. to } 6 \text { p.m. } \\
\text { for weekdays and 9:30 a.m. to 11:30 a.m. and } 3 \text { p.m. to } 6 \text { p.m. } \\
\text { for weekends (Yu et al. 2016)) }\end{array}$ & \multicolumn{2}{|c|}{$1: 62$} \\
\hline \multirow[t]{2}{*}{ Night } & Night-time: 7 p.m. to 6 a.m. (Yang et al. 2019) & \multicolumn{2}{|c|}{$0: 246$} \\
\hline & 0: day-time trip, 1 : night-time trip & \multicolumn{2}{|c|}{$1: 262$} \\
\hline
\end{tabular}

\section{Operational conditions}

$\begin{array}{llll}\text { Avg.Speed } & \text { Average speed based on each urban expressway trip } /(\mathrm{km} / \mathrm{h}) & 43.603 & 18.464\end{array}$

$\begin{array}{lll}\text { Std.Speed Standard deviation of speeds based on each urban } 22.449 & 6.106\end{array}$ expressway trip $/(\mathrm{km} / \mathrm{h})$

\section{Roadway characteristics}

Road The roadway on which the driver spends more than $50 \%$ of

Middle ring: 211 the total trip duration

North-South:53

Inner ring: 148

Yan'an: 50

Yixian: 12

Humin: 34

\section{Modeling Results}

Before developing the proposed models, correlation tests were conducted and no significant correlations between the explanatory variables were found. Then, dummy variable transformations have been used for the Road variable, where the Middle Ring Road was set as the reference considering it holds the longest mileage. With the

8 Bayesian inference technique, relationships between the longitudinal driving capability

9 with travel feature, operational conditions, and roadway characteristics were identified.

10 Credible intervals were calculated and used to find the statistically significant variables.

11 Table 4 and 5 have listed the modeling results of BTQR models for longitudinal risk 
exposure indicator $p_{\text {exposure }}$ and longitudinal risk severity indicator $p_{\text {severity }}$ respectively. Estimated coefficients at quantile $0.10,0.25,0.5,0.75,0.95$ were provided. Moreover, the ordinary BTR models were also established for result comparisons.

\subsection{Longitudinal risk exposure model}

The modeling results of longitudinal risk exposure model are shown in Table 4 and Fig. 4. DIC values of the BTQR models are smaller, which indicates that the proposed models are significantly better in model fits than the BTR model. Then, the BTQR models are statistically different, among which the model at quantile 0.5 shows least performance with the highest DIC and the DIC values decrease from median quantile to both sides with better model performances.

As for the explanatory variables, there are a total of six variables found to be significant while some of them hold different significance and estimated coefficients across the conditional quantiles, as shown in Table 4 and Fig. 4. To be specific, Night and Avg.Speed are consistently significant with positive estimates across the varying quantiles, while Duration, Peak, Std.Speed and Road change their levels of significance over the quantiles.

First, both the consistent significant variables are positively related to longitudinal risk exposure with declining effects throughout the quantiles. This indicates that driving during night times would lead to increased longitudinal risk exposure compared with daytimes, and the impact decreases over quantiles. Besides, trips with higher average speed would lead to higher longitudinal risk exposure, however, the effects on exposure increase are larger at lower quantiles compared with the higher quantiles.

As for other variables, Duration is only significant at quantile 0.9 and above with negative correlations; that is to say, the increase of driving time is likely to reduce the exposure. The variable Peak is significant with positive effects before quantile 0.25 and after 0.95 . This reveals that compared with the off-peak, driving during peak hours would increase the longitudinal risk exposure of trips at low/high exposure levels, but has no significant impact on trips at intermediate levels. In addition, in terms of Std.Speed, the variable is negatively associated with declining trend at quantiles from 0.5 to 0.95 . This can be understood as that drivers inclined to keep shorter distances with LV may be more sensitive to the gap and adjust the vehicle speed more frequently, thus appearing higher speed volatility. Moreover, as for roadway characteristics, in comparison with Middle Ring Road, North-South, Inner Ring and Yan'an Road have all showed to have significant and positive effects on longitudinal risk exposure before first quantile: trips on these roads have higher longitudinal risk exposure, among which Yan'an is most influential. Humin only appears significance at the 50th quantile, whose 
1 exposure is lower than trips on Middle Ring.

2 In addition, through the comparisons with BTQR and BTR models, it can be seen 3 that generally the two models have similar significant variable estimates. Specifically, 4 the significance and sign of each variable of the BTR are the same as the median 5 quantile regression. However, compared with the BTR model, the BTQR model reveals 6 a broader and complete view of the variables. For example, some of the insignificant 7 variables of the mean regression are significant at other quantiles, e.g. Duration only 8 appears significant association with longitudinal risk exposure at quantiles beyond 0.9

9 This indicates that for drivers at different capacity levels, the influential factors of 10 longitudinal risk exposure are distinct. As for some of the significant variables, the 11 coefficient estimates are different from the mean regression and may change across the 12 quantiles, which cannot be revealed through the BTR model only.

13 Table 4: Estimation results for BTQR and BTR models of longitudinal risk exposure

\begin{tabular}{|c|c|c|c|c|c|c|c|}
\hline & \multicolumn{6}{|c|}{ BTQR at quantile } & \multirow{2}{*}{ BTR } \\
\hline & 0.10 & 0.25 & 0.50 & 0.75 & 0.90 & 0.95 & \\
\hline \multicolumn{8}{|c|}{ Coefficient Estimates } \\
\hline \multirow[t]{2}{*}{ Intercept } & 0.009 & $0.312 *$ & $0.628 *$ & $0.749 *$ & $0.883^{*}$ & $0.910 *$ & $0.571^{*}$ \\
\hline & {$[-0.091,0.113]$} & {$[0.203,0.418]$} & {$[0.525,0.723]$} & {$[0.667,0.835]$} & {$[0.802,0.955]$} & {$[0.861,0.957]$} & {$[0.468,0.676]$} \\
\hline \multirow[t]{2}{*}{ Duration } & 0.145 & 0.030 & 0.032 & -0.078 & $-0.205^{*}$ & $-0.214^{*}$ & -0.056 \\
\hline & {$[-0.001,0.288]$} & {$[-0.174,0.222]$} & {$[-0.139,0.184]$} & {$[-0.194,0.041]$} & {$[-0.318,-0.089]$} & {$[-0.314,-0.120]$} & {$[-0.226,0.114]$} \\
\hline \multirow[t]{2}{*}{ Peak } & $0.089 *$ & $0.066^{*}$ & 0.045 & 0.037 & 0.031 & $0.050 *$ & $0.054^{*}$ \\
\hline & {$[0.043,0.136]$} & {$[0.014,0.119]$} & {$[-0.001,0.0899]$} & {$[-0.001,0.076]$} & {$[-0.010,0.075]$} & {$[0.016,0.081]$} & {$[0.009,0.101]$} \\
\hline \multirow[t]{2}{*}{ Night } & $0.116^{*}$ & $0.102 *$ & $0.070 *$ & $0.047^{*}$ & $0.035^{*}$ & $0.021 *$ & $0.072^{*}$ \\
\hline & {$[0.083,0.150]$} & {$[0.068,0.135]$} & {$[0.041,0.099]$} & {$[0.023,0.071]$} & {$[0.014,0.056]$} & {$[0.001,0.043]$} & {$[0.042,0.102]$} \\
\hline \multirow[t]{2}{*}{ Avg.Speed } & $0.005^{*}$ & $0.004 *$ & $0.002 *$ & $0.001 *$ & $0.001 *$ & $0.001 *$ & $0.003 *$ \\
\hline & {$[0.004,0.006]$} & {$[0.003,0.005]$} & {$[0.001,0.003]$} & {$[0,0.002]$} & {$[0,0.002]$} & {$[0,0.002]$} & {$[0.002,0.004]$} \\
\hline \multirow[t]{2}{*}{ Std.Speed } & 0 & -0.001 & $-0.004 *$ & $-0.003 *$ & $-0.003 *$ & $-0.003^{*}$ & $-0.002 *$ \\
\hline & {$[-0.003,0.002]$} & {$[-0.004,0.002]$} & {$[-0.006,-0.001]$} & {$[-0.006,-0.001]$} & {$[-0.005,-0.001]$} & {$[-0.004,-0.001]$} & {$[-0.005,0]$} \\
\hline Road(Nort & $0.153^{*}$ & 0.041 & -0.004 & 0.006 & -0.014 & -0.017 & -0.048 \\
\hline h-South) & {$[0.096,0.210]$} & {$[-0.016,0.101]$} & {$[-0.054,0.046]$} & {$[-0.035,0.043]$} & {$[-0.050,0.024]$} & {$[-0.053,0.017]$} & {$[-0.101,0.006]$} \\
\hline Road(Inne & $0.123^{*}$ & $0.070 *$ & 0.037 & 0.044 & 0.030 & 0.027 & -0.013 \\
\hline r ring) & {$[0.059,0.186]$} & {$[0.009,0.133]$} & {$[-0.013,0.089]$} & {$[-0.002,0.089]$} & {$[-0.009,0.067]$} & {$[-0.008,0.058]$} & {$[-0.051,0.025]$} \\
\hline Road(Yan' & $0.257^{*}$ & $0.138 *$ & 0.009 & 0.040 & 0.033 & 0.027 & 0.031 \\
\hline an) & {$[0.162,0.345]$} & {$[0.075,0.203]$} & {$[-0.046,0.068]$} & {$[-0.023,0.093]$} & {$[-0.017,0.082]$} & {$[-0.008,0.061]$} & {$[-0.028,0.090]$} \\
\hline Road(Yixi & 0.081 & -0.018 & -0.054 & 0.066 & 0.050 & 0.047 & -0.047 \\
\hline an) & {$[-0.046,0.199]$} & {$[-0.119,0.074]$} & {$[-0.186,0.099]$} & {$[-0.026,0.159]$} & {$[-0.024,0.128]$} & {$[-0.010,0.116]$} & {$[-0.141,0.046]$} \\
\hline Road(Hu & -0.002 & -0.074 & $-0.096^{*}$ & -0.001 & -0.004 & 0.031 & $-0.087^{*}$ \\
\hline $\operatorname{min)}$ & {$[-0.089,0.079]$} & {$[-0.150,0.001]$} & {$[-0.189,-0.006]$} & {$[-0.070,0.058]$} & {$[-0.066,0.064]$} & {$[-0.019,0.079]$} & {$[-0.153,-0.021]$} \\
\hline \multicolumn{8}{|c|}{ Goodness of Fit } \\
\hline DIC & -1379 & -1176 & -1124 & -1223 & -1597 & -2247 & -1045 \\
\hline
\end{tabular}

14 Note: * Significant at the $95 \%$ level, inside $[$,$] is the 95 \%$ BCI 

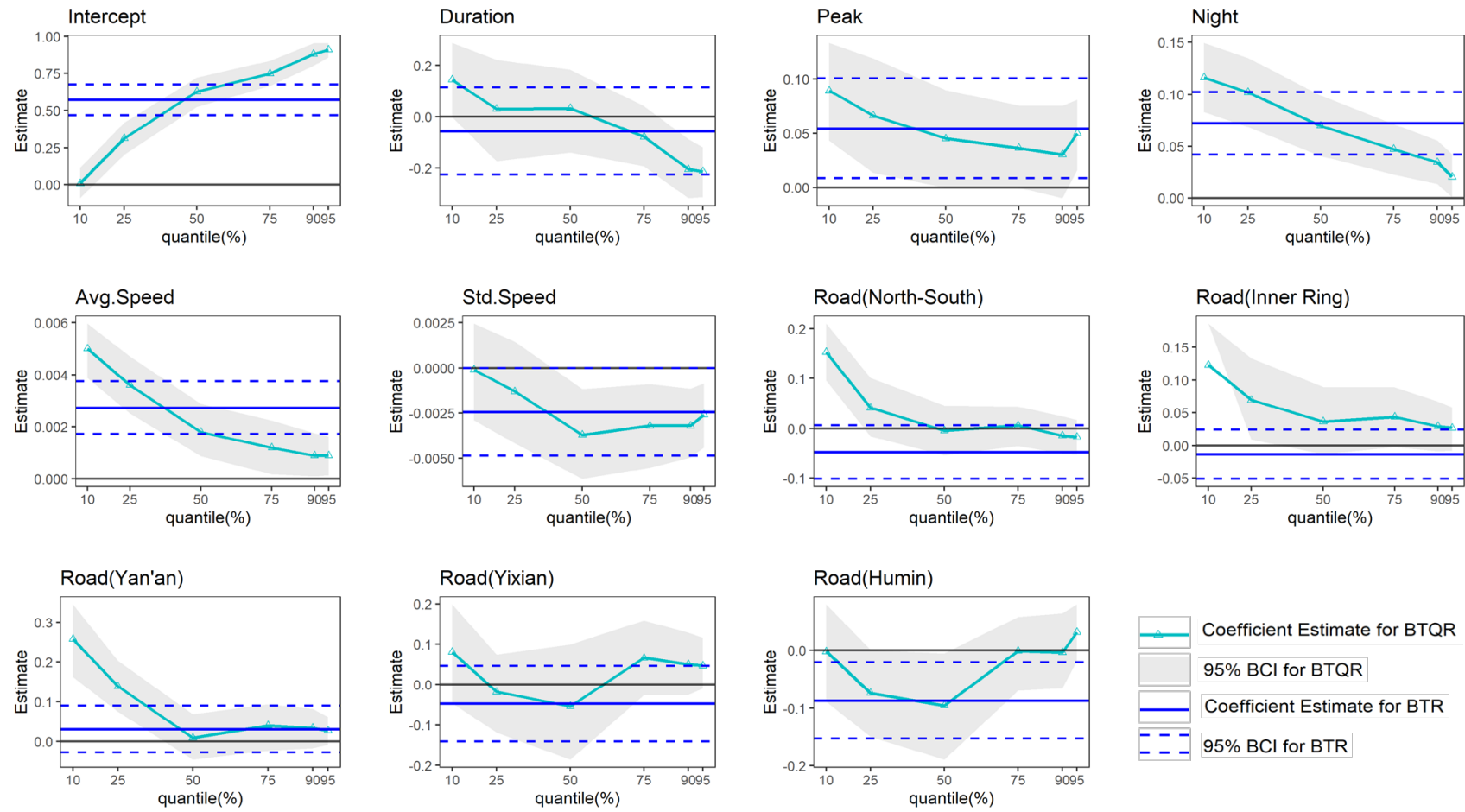

Fig. 4. Quantile plots for coefficient estimates of longitudinal risk exposure

\subsection{Longitudinal risk severity model}

The modeling results of longitudinal risk severity are shown in Table 5 and Fig.5.

5

6 Similar to the risk exposure model, the DIC value of BTR model is the highest, indicating that the proposed BTQR models are significantly better than BTR model according to the DIC. Among the BTQR models, the DIC values reach highest at quantile 0.5 and decline to both sides, indicating that models at higher or lower quantiles performs better than median quantile.

As for the explanatory variables, the BTQR models have the same consistently positive significant variables Night, Avg.Speed and Road have revealed consistently significance with positive estimates through all the quantiles, while Duration and Peak change substantially over the quantiles, and the speed volatility effect characterized by Std.Speed has no impacts on the severity.

First, both consistent significant variables are positively related to longitudinal risk severity throughout the quantiles. The coefficient estimate of Night shows an upward trend, while Avg.Speed holds more stable effects over the quantiles. This indicates that driving during night times have larger probability to be exposed to more severe longitudinal risk compared with daytimes, and the impact increases with the quantile increase. Besides, trips with higher average speed would lead to higher longitudinal risk degree with stable impacts among all the quantiles. 
As for the other variables, Duration is only significant at 0.95 quantile with a negative sign, which indicates that the increase of driving duration would reduce the longitudinal risk severity. The Peak variable is significant with increasing effects except for the 0.25 quantile. This reveals that compared to off-peak, driving during peak hours are likely to have higher risk severity levels. Moreover, in the case of roadway characteristics, trips on Middle Ring Road seem to hold the highest severity, since all significant variable estimates are negative and the trend of effect increases, implying that the decrease of longitudinal risk severity at higher quantiles is larger. North-South Road is significant through all quantiles, Inner Ring and Humin Road at most quantiles, while Yan'an and Yixian Road at certain quantiles.

Furthermore, compared with the BTR model, significant variable estimates in the BTQR model are similar. And no surprisingly, the variable significance and trend of BTR are the same as the median quantile regression. However, the BTQR model reveals 14 a broader and complete view of the variables. For example, some of the insignificant variables of the mean regression are of significance at other quantiles, e.g. Yan'an and Yixian Road only appears significant association to longitudinal risk severity from 17 median to third quantile, implying that for drivers with different capacity, the capacity 18 influential factors are distinct. As for some of the significant variables, the coefficient 19 estimates are different from the mean regression and may change across the quantiles,
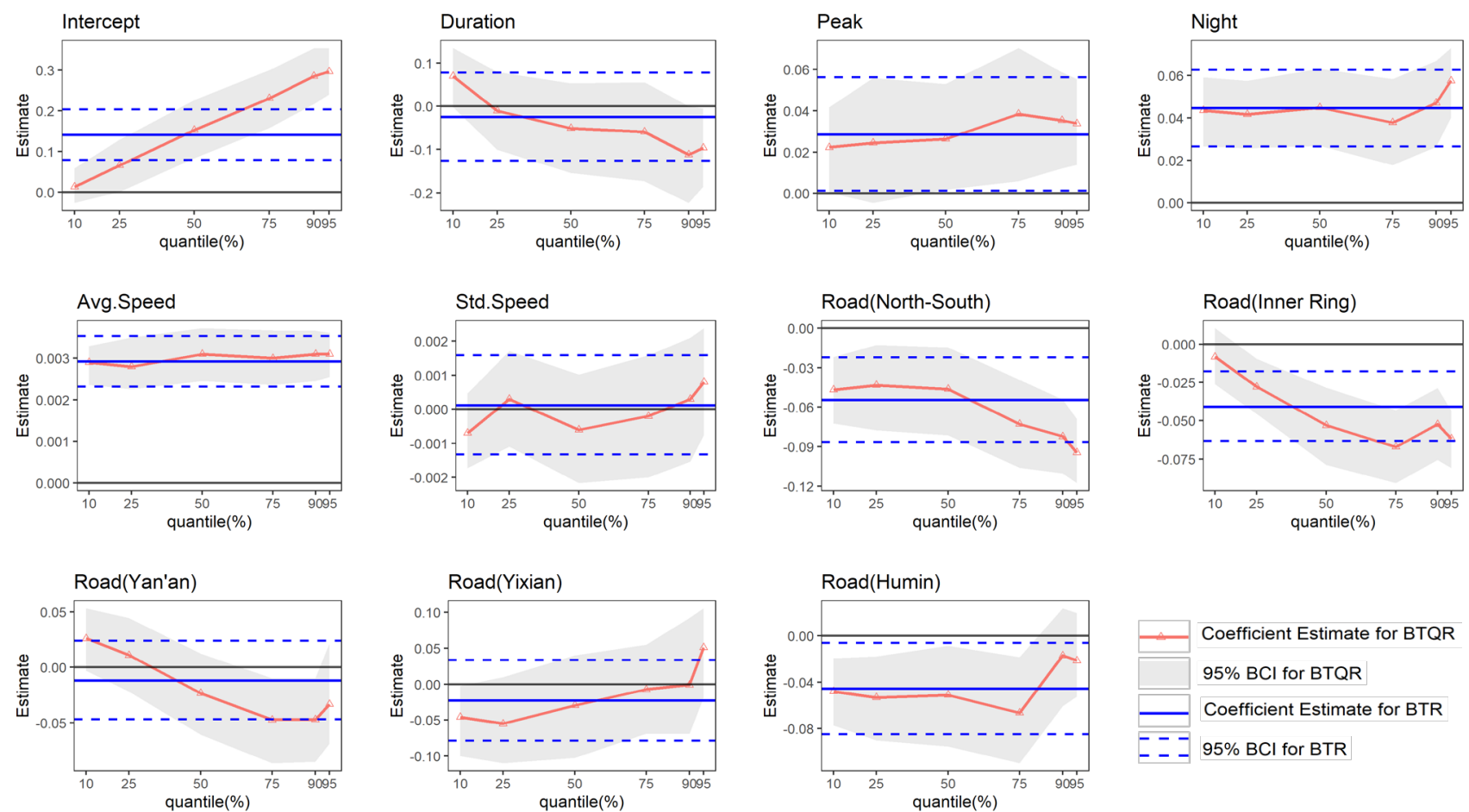

Fig. 5. Quantile plots for coefficient estimates of longitudinal risk severity model 
Table 5: Estimation results for BTQR and BTR models of longitudinal risk severity

\begin{tabular}{|c|c|c|c|c|c|c|c|}
\hline & \multicolumn{6}{|c|}{ BTQR at quantile } & \multirow{2}{*}{ BTR } \\
\hline & 0.10 & 0.25 & 0.50 & 0.75 & 0.90 & 0.95 & \\
\hline \multicolumn{8}{|c|}{ Coefficient Estimates } \\
\hline Intercept & $\begin{array}{c}0.013 \\
{[-0.026,0.059]}\end{array}$ & $\begin{array}{c}0.066^{*} \\
{[0.001,0.129]}\end{array}$ & $\begin{array}{c}0.153^{*} \\
{[0.083,0.226]}\end{array}$ & $\begin{array}{c}0.231 * \\
{[0.158,0.300]}\end{array}$ & $\begin{array}{c}0.285^{*} \\
{[0.216,0.353]}\end{array}$ & $\begin{array}{c}0.297^{*} \\
{[0.240,0.354]}\end{array}$ & $\begin{array}{c}0.1407^{*} \\
{[0.079,0.203]}\end{array}$ \\
\hline Duration & $\begin{array}{c}0.070 \\
{[-0.002,0.135]}\end{array}$ & $\begin{array}{c}-0.010 \\
{[-0.100,0.080]}\end{array}$ & $\begin{array}{c}-0.051 \\
{[-0.154,0.053]}\end{array}$ & $\begin{array}{c}-0.059 \\
{[-0.173,0.055]}\end{array}$ & $\begin{array}{c}-0.112 \\
{[-0.223,0]}\end{array}$ & $\begin{array}{c}-0.096^{*} \\
{[-0.186,-0.005]}\end{array}$ & $\begin{array}{c}-0.025 \\
{[-0.126,0.077]}\end{array}$ \\
\hline Peak & $\begin{array}{c}0.022^{*} \\
{[0,0.042]}\end{array}$ & $\begin{array}{c}0.025 \\
{[-0.005,0.056]}\end{array}$ & $\begin{array}{c}0.026^{*} \\
{[0.002,0.053]}\end{array}$ & $\begin{array}{c}0.038^{*} \\
{[0.006,0.070]}\end{array}$ & $\begin{array}{c}0.035^{*} \\
{[0.012,0.059]}\end{array}$ & $\begin{array}{c}0.034 * \\
{[0.014,0.055]}\end{array}$ & $\begin{array}{c}0.029^{*} \\
{[0.001,0.056]}\end{array}$ \\
\hline Night & $\begin{array}{c}0.044^{*} \\
{[0.027,0.059]}\end{array}$ & $\begin{array}{c}0.042 * \\
{[0.026,0.057]}\end{array}$ & $\begin{array}{c}0.045^{*} \\
{[0.027,0.063]}\end{array}$ & $\begin{array}{c}0.038^{*} \\
{[0.018,0.058]}\end{array}$ & $\begin{array}{c}0.047^{*} \\
{[0.026,0.067]}\end{array}$ & $\begin{array}{c}0.058^{*} \\
{[0.040,0.073]}\end{array}$ & $\begin{array}{c}0.045^{*} \\
{[0.027,0.063]}\end{array}$ \\
\hline Avg.Speed & $\begin{array}{c}0.003^{*} \\
{[0.002,0.003]}\end{array}$ & $\begin{array}{c}0.003^{*} \\
{[0.002,0.004]}\end{array}$ & $\begin{array}{c}0.003^{*} \\
{[0.003,0.004]}\end{array}$ & $\begin{array}{c}0.003 * \\
{[0.003,0.004]}\end{array}$ & $\begin{array}{c}0.003 * \\
{[0.003,0.004]}\end{array}$ & $\begin{array}{c}0.003 * \\
{[0.003,0.004]}\end{array}$ & $\begin{array}{c}0.003^{*} \\
{[0.002,0.004]}\end{array}$ \\
\hline Std.Speed & {$\left[\begin{array}{c}-0.001 \\
{[-0.002,0.001]}\end{array}\right.$} & $\begin{array}{c}0 \\
{[-0.001,0.002]}\end{array}$ & $\begin{array}{c}-0.001 \\
{[-0.002,0.001]}\end{array}$ & $\begin{array}{c}-0.0002 \\
{[-0.002,0.002]}\end{array}$ & $\begin{array}{c}0 \\
{[-0.002,0.002]}\end{array}$ & $\begin{array}{c}0.001 \\
{[-0.001,0.002]}\end{array}$ & $\begin{array}{c}0 \\
{[-0.001,0.002]}\end{array}$ \\
\hline $\begin{array}{c}\text { Road(Nort } \\
\text { h-South) }\end{array}$ & {$\left[\begin{array}{c}-0.047^{*} \\
{[-0.072,-0.022]}\end{array}\right.$} & $\begin{array}{c}-0.043^{*} \\
{[-0.077,-0.013]}\end{array}$ & $\begin{array}{c}-0.046^{*} \\
{[-0.081,-0.015]}\end{array}$ & $\begin{array}{c}-0.073^{*} \\
{[-0.106,-0.039]}\end{array}$ & $\begin{array}{c}-0.082 * \\
{[-0.110,-0.054]}\end{array}$ & $\begin{array}{c}-0.095^{*} \\
{[-0.118,-0.069]}\end{array}$ & $\begin{array}{c}-0.055^{*} \\
{[-0.087,-0.022]}\end{array}$ \\
\hline $\begin{array}{c}\text { Road(Inne } \\
\text { r ring) }\end{array}$ & {$\left[\begin{array}{c}-0.008 \\
-0.026,0.010]\end{array}\right.$} & $\begin{array}{c}-0.028^{*} \\
{[-0.046,-0.010]}\end{array}$ & $\begin{array}{c}-0.053 * \\
{[-0.079,-0.029]}\end{array}$ & $\begin{array}{c}-0.067^{*} \\
{[-0.091,-0.043]}\end{array}$ & $\begin{array}{c}-0.052 * \\
{[-0.076,-0.029]}\end{array}$ & $\begin{array}{c}-0.062 * \\
{[-0.081,-0.044]}\end{array}$ & $\begin{array}{c}-0.041^{*} \\
{[-0.0632,-0.0179]}\end{array}$ \\
\hline $\begin{array}{c}\text { Road(Yan' } \\
\text { an) }\end{array}$ & {$\left[\begin{array}{c}0.026 \\
{[-0.003,0.053]}\end{array}\right.$} & $\begin{array}{c}0.011 \\
{[-0.022,0.044]}\end{array}$ & $\begin{array}{c}-0.023 \\
{[-0.061,0.012]}\end{array}$ & $\begin{array}{c}-0.047^{*} \\
{[-0.086,-0.010]}\end{array}$ & $\begin{array}{c}-0.047^{*} \\
{[-0.085,-0.010]}\end{array}$ & $\begin{array}{c}-0.033 \\
{[-0.069,0.021]}\end{array}$ & $\begin{array}{c}-0.012 \\
{[-0.047,0.024]}\end{array}$ \\
\hline $\begin{array}{l}\operatorname{Road}(Y i x i \\
\text { an) }\end{array}$ & $\begin{array}{c}-0.046^{*} \\
{[-0.10,-0.002]}\end{array}$ & $\begin{array}{c}-0.055 \\
{[-0.110,0.010]}\end{array}$ & $\begin{array}{c}-0.030 \\
{[-0.103,0.040]}\end{array}$ & $\begin{array}{c}-0.007 \\
{[-0.069,0.055]}\end{array}$ & $\begin{array}{c}-0.001 \\
{[-0.069,0.092]}\end{array}$ & $\begin{array}{c}0.051 \\
{[-0.019,0.106]}\end{array}$ & $\begin{array}{c}-0.023 \\
{[-0.079,0.034]}\end{array}$ \\
\hline $\begin{array}{l}\operatorname{Road}(\mathrm{Hu} \\
\min )\end{array}$ & $\begin{array}{c}-0.048^{*} \\
{[-0.077,-0.019]}\end{array}$ & $\begin{array}{c}-0.053 * \\
{[-0.090,-0.018]}\end{array}$ & $\begin{array}{c}-0.051^{*} \\
{[-0.096,-0.009]}\end{array}$ & $\begin{array}{c}-0.067^{*} \\
{[-0.110,-0.019]}\end{array}$ & $\begin{array}{c}-0.017 \\
{[-0.061,0.024]}\end{array}$ & $\begin{array}{c}-0.021 \\
{[-0.052,0.020]}\end{array}$ & $\begin{array}{c}-0.046 \\
{[-0.085,-0.006]}\end{array}$ \\
\hline Goodness o & of Fit & & & & & & \\
\hline DIC & -5841 & -4053 & -3601 & -3951 & -5123 & -7202 & -3385 \\
\hline
\end{tabular}

2 Note: * Significant at the $95 \%$ level, inside [, ] is the $95 \%$ BCI

3

\subsection{Model comparison}

To further explore the impacts of the explanatory variables on longitudinal driving safety, comparisons of the BTQR model for longitudinal risk exposure and severity were conducted. First, the similarities lie in that both sets of models are better than ordinary BTR models according to DIC values, and have the same consistently positive significant variables Night and Avg.Speed, and that Duration is only negatively and significantly related to both indicators at high quantiles.

However, there are also differences in variable significance and coefficient estimates between the models. In the case variable significance, the significance of Std.Speed is only observed in the longitudinal risk exposure model. Then, Peak and roadway characteristics are significant at different quantiles in the two models. As for 
coefficient estimates, the differences consist in the positivity or negativity and the trends across the quantiles. To begin with, although the effect of Peak and Night at significant quantiles in both models are positive, it declines in the longitudinal risk exposure model while the impacts showing an increasing trend in the severity model. Then, the impact of Avg.Speed on longitudinal risk exposure declines over quantiles, while it remains stable in the severity model. Additionally, the effects of roadway characteristics on the two indicators are quite different. Compared with Middle Ring Road, other roadways expect Yixian Road have significant and positive effects on longitudinal risk exposure before median quantile, while roadway choice is of significance with negative impacts on longitudinal risk severity throughout the quantiles, indicating that trips on Middle Ring Road seem to have highest severity.

\section{Case Study}

In this section, the model application scenarios for the drivers' longitudinal driving capability assessment is introduced through a case study. To begin with, with the above mentioned models and the known travel, operational and roadway characteristics, the observed longitudinal risk exposure $p_{\text {exposure }}$ and the longitudinal risk severity $p_{\text {severity }}$ can be calculated and their positions among the sampled data can be further identified. The observed outcomes for specific trips can be compared with different intervals. For instance, the capability can be ranked at $\left[0,10^{\text {th }}\right),\left[25^{\text {th }}, 50^{\text {th }}\right),\left[75^{\text {th }}, 90^{\text {th }}\right)$, etc. Since $p_{\text {exposure }}$ refers to the proportion of the total driving duration under the close following conditions, a higher quantile of this value indicates a higher tendency of the driver keeping a shorter and unsafe distance with a LV throughout the trip, which means a more aggressive driving style. Likewise, $p_{\text {severity }}$ expresses the entity of the longitudinal distance lower than the safety threshold during a whole trip. The higher quantile of this value can be seen as the driver would maintain misjudgments of gaps thus following closely or response too slowly for a cut-in vehicle, the higher the longitudinal risk and the lower the driving skill.

In order to demonstrate how to use the developed models to quantify driving capability, three out-of-sample trips were used as examples. Table 6 illustrates the longitudinal driving capability of the three trips. Taken Trip 1 as an example, both longitudinal risk exposure and severity are located in $\left[25^{\text {th }}, 50^{\text {th }}\right)$ which is a moderate quantile level. This indicates that during the trip the driver reveals a moderate driving style and skill in groups; that is to say, the driver has a relative low tendency of keeping a short distance from a LV, and the gap between the realistic distance and the minimum 
1 safe longitudinal distance is relatively close even if the distance does not reach the 2 safety threshold. As for Trip 2, the capability indicators of exposure and severity fall in $3 \quad\left[75^{\text {th }}, 90^{\text {th }}\right)$ and $\left[50^{\text {th }}, 75^{\text {th }}\right)$ respectively, indicating that the driving performance is of high 4 aggression and below average driving skill among the group. While for Trip 3, the 5 exposure and severity indicators locate in $\left[75^{\text {th }}, 90^{\text {th }}\right)$ and $\left[25^{\text {th }}, 50^{\text {th }}\right)$ respectively, 6 implying that compared with the majority drivers, the driver behaved aggressively but 7 tending to maintain a relatively moderate longitudinal distance to the LV during the trip.

Table 6: Case studies assessment results of longitudinal driving capability

\begin{tabular}{|c|c|c|c|c|}
\hline & & Trip 1 & Trip 2 & Trip 3 \\
\hline \multicolumn{2}{|c|}{ Duration (h) } & 0.134 & 0.450 & 0.109 \\
\hline \multicolumn{2}{|c|}{ Avg.Speed $(\mathrm{km} / \mathrm{h})$} & 58.349 & 44.227 & 47.499 \\
\hline \multicolumn{2}{|c|}{ Std.Speed $(\mathrm{km} / \mathrm{h})$} & 20.527 & 28.834 & 35.352 \\
\hline \multicolumn{2}{|l|}{ Peak } & 0 & 0 & 1 \\
\hline \multicolumn{2}{|l|}{ Night } & 1 & 1 & 0 \\
\hline \multicolumn{2}{|l|}{ Road } & Middle Ring & Middle Ring & North-South \\
\hline \multicolumn{2}{|l|}{$p_{\text {exposure }}$} & 0.764 & 0.756 & 0.741 \\
\hline \multirow{6}{*}{$\begin{array}{c}\text { Estimate } p_{\text {exposure }} \\
\text { at quantile }\end{array}$} & 0.10 & 0.554 & 0.480 & 0.347 \\
\hline & 0.25 & 0.671 & 0.583 & 0.507 \\
\hline & 0.50 & 0.771 & 0.706 & 0.633 \\
\hline & 0.75 & 0.836 & 0.755 & 0.722 \\
\hline & 0.90 & 0.906 & 0.793 & 0.820 \\
\hline & 0.95 & 0.926 & 0.815 & 0.886 \\
\hline \multicolumn{2}{|c|}{$\begin{array}{c}\text { Estimate longitudinal risk } \\
\text { exposure level }\end{array}$} & {$\left[25^{\text {th }}, 50^{\text {th }}\right)$} & {$\left[75^{\text {th }}, 90^{\text {th }}\right)$} & {$\left[75^{\text {th }}, 90^{\text {th }}\right)$} \\
\hline \multicolumn{2}{|l|}{$p_{\text {severity }}$} & 0.305 & 0.295 & 0.190 \\
\hline \multirow{6}{*}{$\begin{array}{c}\text { Estimate } p_{\text {severity }} \\
\text { at quantile }\end{array}$} & 0.10 & 0.220 & 0.167 & 0.109 \\
\hline & 0.25 & 0.278 & 0.209 & 0.191 \\
\hline & 0.50 & 0.361 & 0.264 & 0.254 \\
\hline & 0.75 & 0.432 & 0.338 & 0.325 \\
\hline & 0.90 & 0.503 & 0.396 & 0.383 \\
\hline & 0.95 & 0.536 & 0.438 & 0.399 \\
\hline \multicolumn{2}{|c|}{$\begin{array}{c}\text { Estimate longitudinal risk } \\
\text { severity level }\end{array}$} & {$\left[25^{\text {th }}, 50^{\text {th }}\right)$} & {$\left[50^{\text {th }}, 75^{\text {th }}\right)$} & {$\left[10^{\text {th }}, 25^{\text {th }}\right)$} \\
\hline
\end{tabular}

Meanwhile, it can be found that the developed models are capable of differentiating trips with heterogeneous traffic scenarios. To be more specific, Trip 1 and Trip 2 hold close $p_{\text {exposure }}$ and $p_{\text {severity }}$ values as well as same travel time and roadway characteristics, but there are differences in the longitudinal driving capacity levels of 13 both driving style and skill because of diverse operational characteristics. This is the 14 advantage of the proposed approach through considering the confounding impacts of 
road, traffic, and environment factors when evaluate the driving capability.

\section{Discussion and Conclusion}

Driving capability assessment has become an important area of research due to the severe safety problem and massive demand for safety improvement. However, traditional assessment approaches such as driving license exams and vehicle insurance quotes have their limitations (e.g. the road test modules of driving license exams are too simple to reflecting naturalistic driving capability) due to lack of including streaming data environment. Therefore, they normally failed to meet the needs of evaluating relative driving capability. Moreover, most previous studies conducted assessments based on fragmented and specific driving events, which ignored the most complex but ubiquitous task transition periods. In addition, the indicators utilized failed to take account of the heterogeneous traffic scenarios and cannot meet the needs of driving capability ranking, while the algorithms were unavailable for both classification and interpretation.

In this study, a quantified driving capability assessment approach was developed to address the above-mentioned problems with RSS-based indicators and BTQR models. RSS-based driving capability indicators were established from the perspectives of longitudinal risk exposure and severity whilst BTQR models were developed to determine the ranked thresholds of both indicators and explore relationships between driving capability with travel, operational and roadway characteristics. Empirical analyses of longitudinal driving capability were conducted based on vehicle kinematic data from the Shanghai urban expressway system.

From the modeling results, it can be found that BTQR models have revealed a broader and complete view of the trip characteristics compared with BTR models. More specifically, nighttime driving and higher average speed would lead to larger longitudinal collision risk and its severity. Besides, the BTQR models have provided varying factors significances among different quantile levels, for instance, driving duration is only negatively and significantly related with both driving capability indicators at very high quantiles, implying that drivers possessing high longitudinal risk and severity may keep a more stable mindset during a long driving process.

Then, through the comparisons of BTQR based longitudinal risk exposure and severity models, similarities and difference between the models were observed. The similarities lie in that nighttime driving and average speed are found to be consistently significant through all the quantiles, indicating that nighttime driving or higher average speed would lead to higher longitudinal risk exposure and severity of the trip; and that 
driving duration is significant at high quantiles, as mentioned above. The differences also lie in variable significance and coefficient estimates; e.g., the significance of speed volatility is only observed in the exposure model, and peak hour driving and roadway characteristics are significant at different quantiles in the two models. Besides, it is interesting to find that speed volatility is negatively associated with longitudinal risk exposure from median quantile, which can be understood as that drivers inclined to keep shorter longitudinal distances with LV may be more sensitive to the gap and adjust the vehicle speed more frequently, thus appearing higher speed volatility.

As for the model application scenarios, with captured trip characteristics, the relative positions of observed driving capability indicators can be identified through the developed capability evaluation models. A higher quantile level of longitudinal risk exposure can be seen as a higher tendency of close following, which means a more aggressive driving style. Likewise, a higher quantile level of longitudinal risk severity indicates that the driver would maintain misjudgment of gaps thus following closely or responding too slowly for a cut-in vehicle, which implies lower driving skill with a higher longitudinal collision risk and lower safety awareness. Therefore, the longitudinal safe keeping capability can be roughly categorized into five groups, as the skill-style plane shown in Fig. 6:

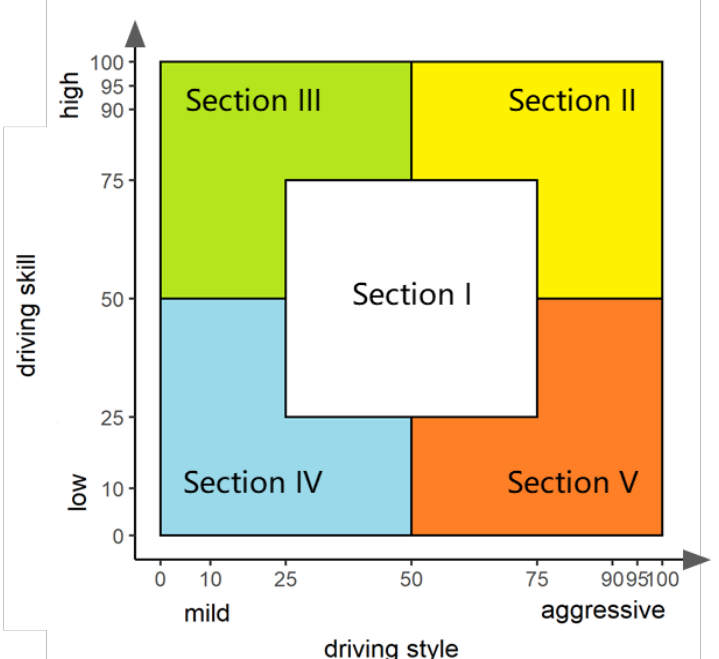

Fig. 6. skill-style plane with quantile levels (\%)

(a) Section I: moderate style and skill, with the quantile levels of both driving capability located in moderate intervals;

(b) Section II: aggressive style and high skill, revealing that the driver behaves aggressively but controls the longitudinal distance skillfully, used to keeping a slightly shorter distance from LV;

(c) Section III: mild style and high skill, revealing that the driver keeps moderate and safe distance from LV and the gap between the realistic distance and the minimum 
safe longitudinal distance is relatively close even if the distance does not reach the safety threshold;

(d) Section IV: mild style and low skill, revealing that the driver keeps a longer distance than the threshold most of the time, but the gap between ego vehicle and LV is large enough for another vehicle to cut in and trigger a safety critical event. Thus, a driver with these characteristics still needs to improve his or her capability to keep a both safe and moderate longitudinal distance and avoid being cut-in frequently;

(e) Section V: aggressive style and low skill, revealing that the driver holds pool driving capability and drives in a risky way, with lack of longitudinal distance recognition and control.

It can be seen from the above analysis that driving capability cannot be summarized by one of the indicators, of which the complete view is given by the combination of style and skill. Besides, it is interesting to find that the same longitudinal risk exposure or severity value may represent different capability levels due to diverse traffic conditions, which indicates the model adaptability to various scenarios.

Finally, the developed analysis technique can further be extended and applied in different aspects. First, the similar analysis approach can be used to for lateral driving capability assessment. Then, the analysis results could shed some lights on commercial fleet safety management through driving capability ranking and feedbacks. For instance, given the large installed coverage of environmental monitoring instruments for commercial vehicles, the required data to evaluate the driving capability can be collected. Then, the trip-level capability rankings could be obtained based upon the models, which can be applied to identify the risky drivers and drivers with low capabilities. Since crash is a rare event and crash occurrence has complex influencing factors, compared with traditional crash-based driving capability assessment method, the proposed approach takes objective trip characteristics into consideration and holds the advantages of identifying risky drivers in advance to the crash occurrences. Besides, different driving capabilities can be ranked objectively as the case study showed. Moreover, considering the hot topic of autonomous vehicle (AV) safety evaluation, the AVs' driving performance can be compared with the human drivers using the proposed method. Based on the developed BTQR models and AV road testing data, the trip-level AV driving capability rankings compared to the human drivers can be obtained. The analysis could be used to enhance the AV's human-alike features and help to recognize critical scenarios where AVs perform poorly. In addition to practical applications in fleet safety management and AV performance evaluations, the developed models can also have some implications for policy making. For instance, the framework of driving license management could be redesigned. With the ranked driving capabilities, the 
1 driving re-education programs and the safe driving campaigns can be more focused and 2 targeted. However, there are still several limitations. As for the employed dataset, only 3 structured data (kinematic, GPS, and radar) were used where future analyses with richer 4 data sources (e.g. video) can take more environment factors (e.g. weather) and invehicle behaviors into account to further improve the model.

\section{Acknowledgments}

This study was joint supported by National Key R\&D Program of China (No.2019YFB1600703) and Chinese National Natural Science Foundation (NSFC) under Grant No.71771174.

\section{Declarations of Interest}

None.

\section{References}

Alhamzawi, R., 2018. Brq: An $\mathrm{r}$ package for bayesian quantile regression. Working Paper URL: https://cran. r-project. org/web/packages/Brq/Brq. pdf.

Allen, B.L., Shin, B.T., Cooper, P.J., 1978. Analysis of traffic conflicts and collisions.

Elander, J., West, R., French, D., 1993. Behavioral correlates of individual differences in road-traffic crash risk: An examination of methods and findings. Psychological bulletin 113 (2), 279.

Florida DMV, 2019. Florida Driving Test.

Galkin, A., Davidich, N., Filina-Dawidowicz, L., Davidich, Y., 2019. Improving the safety of urban freight deliveries by organization of the transportation process considering driver's state. Transportation research procedia 39, 54-63.

Ghasemzadeh, A., Ahmed, M.M., 2017. Drivers' lane-keeping ability in heavy rain: Preliminary investigation using shrp 2 naturalistic driving study data. Transportation research record 2663 (1), 99-108.

Higgs, B., Abbas, M., Year. A two-step segmentation algorithm for behavioral clustering of naturalistic driving styles. In: Proceedings of the 16th International IEEE Conference on Intelligent Transportation Systems (ITSC 2013), pp. 857-862.

Hydén, C., 1987. The development of a method for traffic safety evaluation: The swedish traffic conflicts technique. Bulletin Lund Institute of Technology, Department (70).

Iida, Y., Uno, N., Itsubo, S., Suganuma, M., Year. Traffic conflict analysis and modeling of lane-changing behavior at weaving section. In: Proceedings of the Proceedings of Infrastructure Planning, pp. 305-308.

Johnson, D.A., Trivedi, M.M., Year. Driving style recognition using a smartphone as a sensor platform. In: Proceedings of the 2011 14th International IEEE Conference on Intelligent Transportation Systems (ITSC), pp. 1609-1615. 
Johnsson, C., Laureshyn, A., De Ceunynck, T., 2018. In search of surrogate safety indicators for vulnerable road users: A review of surrogate safety indicators. Transport Reviews 38 (6), 765785.

Kitajima, S., Takatori, O., Enokida, S., Takeda, K., Katayama, T., 2009. Estimation of driver's dangerous states of rear-end collision based on driver video recorder data and ordinary driving data. Proceedings of automotive engineers of japan. pp. 89-97.

Koenker, 2005. Quantile regression (econometric society monographs; no. 38) Cambridge university press.

Koenker, R., 2017. Quantile regression: 40 years on. Annual Review of Economics 9, 155-176.

Koenker, R., Bassett, G., 1978. Regression quantiles. Econometrica: journal of the Econometric Society, 33-50.

Li, L., Peng, X., Wang, F.-Y., Cao, D., Li, L., 2018. A situation-aware collision avoidance strategy for car-following. IEEE/CAA Journal of Automatica Sinica 5 (5), 1012-1016.

Li, Y., Lu, J., Yang, H., Zhang, W., Liu, Q., 2015. Safety evaluation of car-following behaviors between novices and experienced drivers. Cictp 2015. pp. 2731-2741.

Liu, X., Saat, M.R., Qin, X., Barkan, C.P., 2013. Analysis of us freight-train derailment severity using zero-truncated negative binomial regression and quantile regression. Accident Analysis \& Prevention 59, 87-93.

Malhotra, N., Starkey, N.J., Charlton, S.G., 2017. Driving under the influence of drugs: Perceptions and attitudes of new zealand drivers. Accident Analysis \& Prevention 106, 44-52.

Mapbox, 2018. Open source routing machine.

Martin, A.D., Quinn, K.M., Park, J.H., 2011. Mcmcpack: Markov chain monte carlo in r.

Martinez, C.M., Heucke, M., Wang, F.-Y., Gao, B., Cao, D., 2017. Driving style recognition for intelligent vehicle control and advanced driver assistance: A survey. IEEE Transactions on Intelligent Transportation Systems 19 (3), 666-676.

Meng, A., Siren, A., 2012. Cognitive problems, self-rated changes in driving skills, driving-related discomfort and self-regulation of driving in old drivers. Accident Analysis \& Prevention 49, 322-329.

Merrikhpour, M., Donmez, B., Wang, C., Hayes, B., Grush, B., Year. Associations between drivers' safety records and driving styles: A naturalistic study. In: Proceedings of the Proceedings of the Human Factors and Ergonomics Society Annual Meeting, pp. 1973-1977.

Minderhoud, M.M., Bovy, P.H., 2001. Extended time-to-collision measures for road traffic safety assessment. Accident Analysis \& Prevention 33 (1), 89-97.

National Safety Council, 2017, Injury Facts, 2015 Edition.

Ohta, H., 1993. Individual differences in driving distance headway. Vision in vehicles 4, 91-100.

Qin, X., 2012. Quantile effects of causal factors on crash distributions. Transportation research record 2279 (1), 40-46.

Sagberg, F., Selpi, Bianchi Piccinini, G.F., Engström, J., 2015. A review of research on driving styles and road safety. Human factors 57 (7), 1248-1275.

Shalev-Shwartz, S., Shammah, S., Shashua, A., 2017. On a formal model of safe and scalable self-driving cars. arXiv preprint arXiv:1708.06374.

Singh, S., 2015. Critical reasons for crashes investigated in the national motor vehicle crash causation survey.

Spiegelhalter, D.J., Best, N.G., Carlin, B.P., Van Der Linde, A., 2002. Bayesian measures of model 
complexity and fit. Journal of the royal statistical society: Series b (statistical methodology) 64 (4), 583-639.

Stolwyk, R.J., Charlton, J.L., Triggs, T.J., Iansek, R., Bradshaw, J.L., 2006. Neuropsychological function and driving ability in people with parkinson's disease. Journal of Clinical and Experimental Neuropsychology 28 (6), 898-913.

Tarko, A.P., 2012. Use of crash surrogates and exceedance statistics to estimate road safety. Accident Analysis \& Prevention 45, 230-240.

Tobin, J., 1958. Estimation of relationships for limited dependent variables. Econometrica: journal of the Econometric Society, 24-36.

Van Der Horst, A.R.A., 1991. A time-based analysis of road user behaviour in normal and critical encounters.

Wang, X., Yang, M., Hurwitz, D., 2019. Analysis of cut-in behavior based on naturalistic driving data. Accident Analysis \& Prevention 124, 127-137.

Washington, S., Haque, M.M., Oh, J., Lee, D., 2014. Applying quantile regression for modeling equivalent property damage only crashes to identify accident blackspots. Accident Analysis \& Prevention 66, 136-146.

World Health Organization., 2018. Global status report on road safety 2018: Summary. World Health Organization

Xu, X., Šarić, Ž., 2018. Investigation into interactions between accident consequences and traffic signs: A bayesian bivariate tobit quantile regression approach. Journal of Advanced Transportation 2018.

Yang, D., Xie, K., Ozbay, K., Yang, H., Budnick, N., 2019. Modeling of time-dependent safety performance using anonymized and aggregated smartphone-based dangerous driving event data. Accident Analysis \& Prevention 132, 105286.

Yu, K., Stander, J., 2007. Bayesian analysis of a tobit quantile regression model. Journal of Econometrics 137 (1), 260-276.

Yu, R., Wang, X., Yang, K., Abdel-Aty, M., 2016. Crash risk analysis for shanghai urban expressways: A bayesian semi-parametric modeling approach. Accident Analysis \& Prevention 95, 495-502.

Zheng, L., Ismail, K., Meng, X., 2014a. Freeway safety estimation using extreme value theory approaches: A comparative study. Accident Analysis \& Prevention 62, 32-41.

Zheng, L., Ismail, K., Meng, X., 2014b. Traffic conflict techniques for road safety analysis: Open questions and some insights. Canadian journal of civil engineering 41 (7), 633-641.

Zheng, Z., Ahn, S., Chen, D., Laval, J., 2013. The effects of lane-changing on the immediate follower: Anticipation, relaxation, and change in driver characteristics. Transportation research part C: emerging technologies 26, 367-379. 